\title{
4:1 Silicon Photonic Serializer for Data Center Interconnects Demonstrating 104 Gbaud OOK and PAM4 Transmission
}

Verbist, Jochem; Vanhoecke, Michael; Lillieholm, Mads; Srinivasan, Srinivasan Ashwyn; De Heyn, Peter; Van Campenhout, Joris; Galili, Michael; Oxenløwe, Leif Katsuo; Yin, Xin; Bauwelinck, Johan

Total number of authors:

11

Published in:

Journal of Lightwave Technology

Link to article, DOI:

10.1109/JLT.2019.2897401

Publication date:

2019

Document Version

Peer reviewed version

Link back to DTU Orbit

Citation (APA):

Verbist, J., Vanhoecke, M., Lillieholm, M., Srinivasan, S. A., De Heyn, P., Van Campenhout, J., Galili, M., Oxenløwe, L. K., Yin, X., Bauwelinck, J., \& Roelkens, G. (2019). 4:1 Silicon Photonic Serializer for Data Čenter Interconnects Demonstrating 104 Gbaud OOK and PAM4 Transmission. Journal of Lightwave Technology, 37(5), 1498-1503. https://doi.org/10.1109/JLT.2019.2897401

\section{General rights}

Copyright and moral rights for the publications made accessible in the public portal are retained by the authors and/or other copyright owners and it is a condition of accessing publications that users recognise and abide by the legal requirements associated with these rights.

- Users may download and print one copy of any publication from the public portal for the purpose of private study or research.

- You may not further distribute the material or use it for any profit-making activity or commercial gain

- You may freely distribute the URL identifying the publication in the public portal 


\title{
4:1 Silicon Photonic Serializer for Data Center Interconnects Demonstrating 104 Gbaud OOK and PAM4 Transmission
}

\author{
Jochem Verbist*, Michael Vanhoecke*, Mads Lillieholm, Srinivasan Ashwyn Srinivasan, Peter \\ De Heyn, Joris Van Campenhout, Michael Galili, Leif K. Oxenløwe, Xin Yin, \\ Johan Bauwelinck, and Gunther Roelkens
}

\begin{abstract}
With next-generation optical interconnects for data centers aiming for $0.8 \mathrm{~Tb} / \mathrm{s}$ or $1.6 \mathrm{~Tb} / \mathrm{s}, 100 \mathrm{Gbaud}$ capable transmitters from a single-laser source will become indispensable. However, these lane rates would require bandwidths of $70 \mathrm{GHz}$ or more, doubling the bandwidth requirements of the electrical and optical components with respect to the fastest current generation of optical interconnects running at 53 Gbaud PAM-4. In this paper, we propose an integrated 4:1 optical serializer topology to achieve 104 Gbaud NRZ and PAM-4 transmission using only quarter rate components at the transmitter. We show $104(208) \mathrm{Gbit} / \mathrm{s}$ OOK (PAM4) transmission using four GeSi EAMs over $1 \mathrm{~km}$ of SMF. For 104 Gbaud OOK, clearly open eves are obtained, while for PAM-4 the performance is limited by the non-linear E/O-transfer function of the EAM. However, adding pre-emphasis in the electrical driver or replacing the single EAM with our previously demonstrated optical DAC topology -consisting of two EAMs in parallel with a $90^{\circ}$ phase difference between each- could substantially improve these results. Additionally, we discuss the possibility of a four channel transmitter $(4 \times 208 \mathrm{~Gb} / \mathrm{s})$ from a single mode locked laser, amounting to a $832 \mathrm{~Gb} / \mathrm{s}$ rate based on the current demonstrator.
\end{abstract}

Index Terms -integrated optics, optical interconnects, silicon photonics, waveguide modulators

This paragraph of the first footnote will contain the date on which you submitted your paper for review.

This work was supported in part by SPOC (DNRF123). The authors also thank imec's optical I/O program for the fabrication of the EAMs.

* J.Verbist and M.Vanhoecke contributed equally to this work.

J. Verbist is with the Ghent University-IMEC, IDLab, Department of Information Technology, Ghent 9052, Belgium. He is also with the Ghent University-IMEC, Photonics Research Group, Department of Information Technology, Ghent 9052, Belgium (e-mail: jochem.verbist@ugent.be).He currently works at BiFAST, Ghent 9000, Belgium (e-mail: jochem@bifast.io).

M. Vanhoecke, X. Yin and J. Bauwelinck are with the Ghent UniversityIMEC, IDLab, Department of Information Technology, Ghent 9052, Belgium (e-mail:_michael.vanhoecke@ugent.be;_xin.yin@ugent.be; johan.bauwelinck@ugent.be)

M. Lillieholm, Michael Galili and Leif K. Oxenløwe are with DTU Fotonik, Technical University of Denmark, DK-2800 Kgs. Lyngby, Denmark (e-mail: mads1@fotonik.dtu.dk,mgal@fotonik.dtu.dk,lkox@fotonik.dtu.dk)

A. Srinivasan, P. De Heyn and J. Van Campenhout are with the IMEC, Leuven 3001, Belgium (e-mail: ashwyn.srinivasan@imec.be; Peter.DeHeyn@imec.be; joris.vancampenhout@imec.be).

G. Roelkens is with Ghent University-IMEC, Photonics Research Group, Department of Information Technology, Ghent 9052, Belgium (e-mail: gunther.roelkens@ugent.be)

\section{INTRODUCTION}

A $S$ the bandwidth requirements for data center interconnects (DCI) keep increasing, the current generation of $100 \mathrm{~Gb} / \mathrm{s}$ ( 4 lanes of $25 \mathrm{~Gb} / \mathrm{s}$ NRZ) single-mode fiber links covering the $500 \mathrm{~m}$ to $2 \mathrm{~km}$ spans will need to be upgraded to $(200 \mathrm{~Gb} / \mathrm{s}$ or) $400 \mathrm{~Gb} / \mathrm{s}$ using either (4) 8 lanes of 25 Gbaud PAM-4 or (2) 4 lanes of 50 Gbaud PAM-4 as described by the IEEE 400GBASE standards. For these $100 \mathrm{~Gb} / \mathrm{s} / \lambda$ applications, we have recently demonstrated the first real-time $128 \mathrm{~Gb} / \mathrm{s}$ PAM-4 link using a binary-driven, silicon-based transmitter without the need of any DSP [1]. However, with the first generation of transceivers operating at $100 \mathrm{~Gb} / \mathrm{s} / \lambda$ finding their way to the market in the coming years, research and discussion have already started to shift towards the implementation of the next-generation of optical transceivers operating at $800 \mathrm{~Gb} / \mathrm{s}$ or even $1.6 \mathrm{~Tb} / \mathrm{s}$ [2]. To maintain a low channel count, increasing the rates to $200 \mathrm{~Gb} / \mathrm{s} / \lambda$ per lane schemes would be an elegant solution for these $0.8 \mathrm{~Tb} / \mathrm{s}$ and $1.6 \mathrm{~Tb} / \mathrm{s}$ links.

One option would be to double the amount of bits per symbol again from PAM-4 (2 bit/symbol) to PAM-16 (4 bit/symbol). However, the transition of the NRZ to PAM-4 has already set significant additional requirements on many of the components in the E/O link such as linearity and higher sensitivity (minimally $4.8 \mathrm{~dB}$ due to modulation loss and higher when accounting for any additional penalties due to inter-symbol interference [3]), as well as a need for fast DACs and ADCs. All these specifications have come with a substantial increase in the power budget when compared to the simple OOK schemes. Moving to PAM-16 would burden the optical link budget even further, demanding an additional improvement of at least $7 \mathrm{~dB}$ in receiver sensitivity, as well as a corresponding increase in the effective number of bits (ENOB) of the DACs/ADCs in the link, likely increasing their already large power consumption much further. Therefore, the prospect of implementing a PAM-16 link seems questionable.

A second path to increase the spectral efficiency of links would be to abandon intensity-modulated direct-detection links (IMDD) and shift to optical coherent technology. The additional signal space, by encompassing the quadrature component, and the increased receiver sensitivity, by using a local oscillator, allows doubling the number of bits/symbol to 4 by employing a 16-QAM modulation format. Yet, data 
center operators have been reluctant to adopt these metro and long-haul devices as their significantly higher power consumption makes it challenging to meet the extremely compact form factor requirements. Best in class coherent modules currently require $\sim 20 \mathrm{~W}$ for a $200 \mathrm{Gbit} / \mathrm{s}$ PDM16QAM link using a $4 \times 10.7 \mathrm{~cm}$ CFP2-DCO transceiver module [4]. The additional power consumption of the coherent DSP and the large form factor make IMDD still the preferred solution for data center interconnects.

A last straightforward way to increase the line rate is to double the symbol rate to 100 Gbaud, however the implementation is far more burdensome than the concept. The higher symbol rate will require a significant performance increase of the current generation optical and electrical components, requiring bandwidths of $70 \mathrm{GHz}$ or more. Moreover receiver noise will be higher and jitter tolerance will be lower. The challenge in stepping up the baud rate was revealed by the choice of the standardizing committees to go for PAM-4 modulation over the NRZ format for 400 Gigabit Ethernet DCI.

Despite the challenges, an increase of the symbol rate would be a favored solution for DCI and has been subject of much recent research. A $204 \mathrm{~Gb} / \mathrm{s}$ On-Off keying (OOK) transmitter has been demonstrated using InP platforms for both the optics and electronics in combination with offline DSP [5,6]. Similarly, an EAM integrated with a DFB laser (EADFB) was used to demonstrate $214 \mathrm{~Gb} / \mathrm{s}$ PAM-4 transmission [7]. On silicon, the highest reported intensity modulated transmission has been limited to $168 \mathrm{~Gb} / \mathrm{s}$ PAM-4 using a large multi-electrode traveling-wave Mach-Zehnder modulator (MZM) driven with voltages up to $5 \mathrm{Vpp}$ in combination with extensive Tx-side DSP [8], making it less suitable for use in data centers. In line with our previous work, we move functionality to the optical domain to leverage the performance benefits [1,9] and we focus on a silicon photonic transmitter including a $4: 1$ serializer to overcome the barriers in stepping up the symbol rates in DCI applications.

Over the last two decades, optical serializers (used in optical time division (OTDM) schemes) have attracted much attention as they can generate very high data rate optical transmissions with limited bandwidth electronics [10]. However the need for long integrated optical delays as well as the absence of a practical and low-cost integrated pulsed laser source has prevented the adoption of optical serializers in datacom applications. Nevertheless, remarkable progress has been made in recent years on integrated semiconductor modelocked lasers (MLLs) [11,12]. In particular, the arrival of efficient and low-cost III-V-on-Si MLLs [11] could be an important turning point towards fully integrated Si-based optically serialized transceivers. Silicon Photonics (SiP) would be an ideal platform to integrate MLLs and optical serializers as both devices require long optical time delays, which can be made very compact and with low losses on this platform. Combining a III-V-on-Si laser with the Si multiplexer and a Si modulator would be a promising and cost effective candidate towards realizing 0.8 and $1.6 \mathrm{TbE}$ links, maintaining the low complexity and minimal DSP associated with IMDD links without straining the electronics at the transmitter side.

In this paper, we demonstrate the first silicon modulator capable of generating 104 Gbaud OOK and PAM-4, using four GeSi electro-absorption modulators (EAMs) as an optical serializer. Driving the EAMs with PAM-4, we achieve the highest reported single-wavelength bitrate for a silicon modulator $(208 \mathrm{~Gb} / \mathrm{s})$ in an IMDD link. The compact EAMs $(80 \mu \mathrm{m})$ can be operated without long traveling-wave electrodes and power-consuming terminations (saving transceiver real estate and power) and require only $1.2 \mathrm{Vpp}$ at quarter-rate speeds (26 Gbaud) thanks to the $4: 1$ optical multiplexing. Further improvement is anticipated when the presented topology is enhanced with our $\mathrm{SiPh}$ transmitter demonstrated in [1].

\section{4:1 SERIALIZER ARCHITECTURE AND Silicon PHOtONIC IMPLEMENTATION WITH GESI EAMS}

The topology of the serializer is depicted in Fig. 1. The discussion will be done for a 4 to 1 serializer, but an equivalent approach can be taken for a 2 to 1 structure or any other integer number.

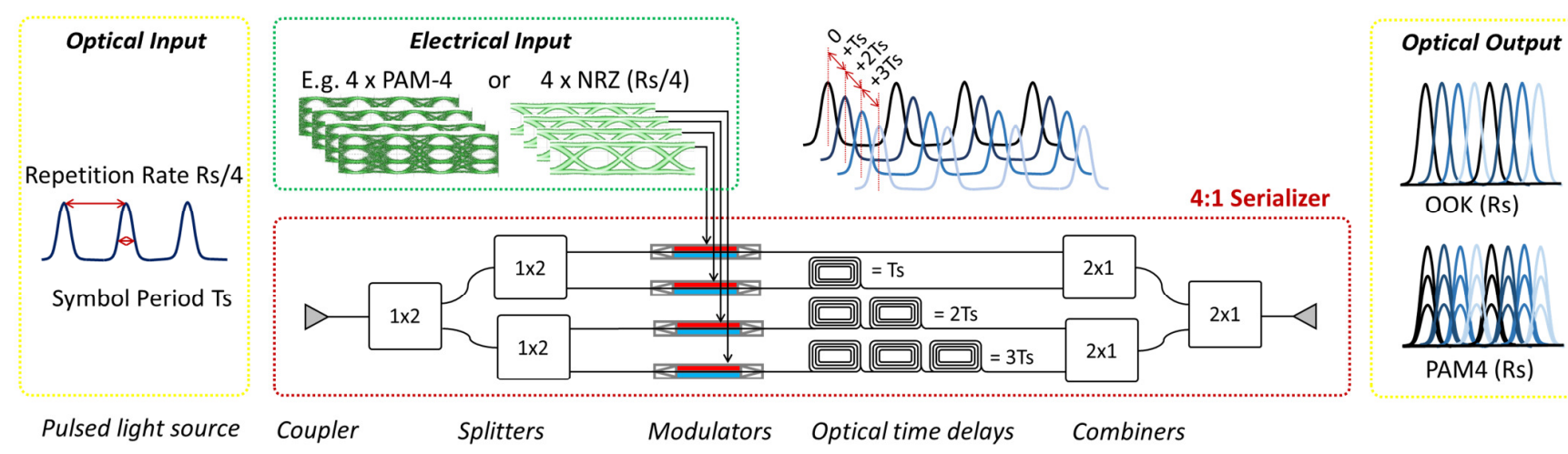

Fig. 1. Operation principle of an optical 4:1 serializer and modulator: For a target symbol rate Rs, a pulsed light source with a pulse width Ts (corresponding to the desired symbol rate) and a repetition rate at the quarter rate $(\mathrm{Rs} / 4)$ enters the optical chip. The light is divided equally over 4 branches where it is intensity modulated at fs $/ 4$ and delayed over 0 to 3 symbol periods. After recombination, a waveform is achieved at the target Rs symbol rate. 


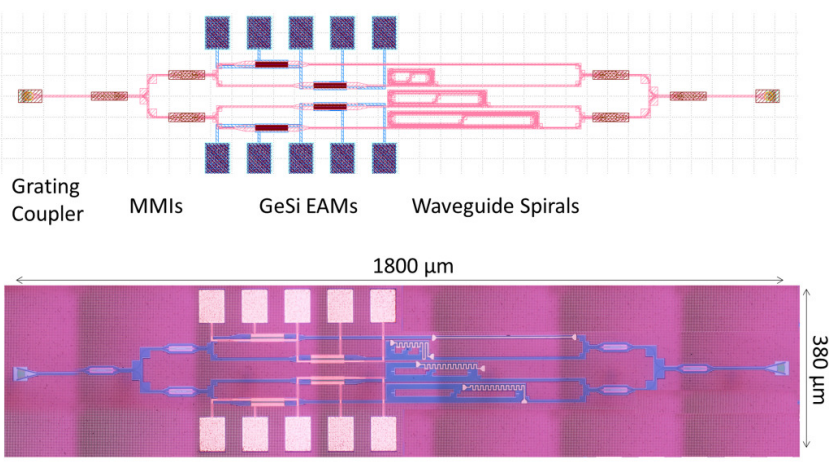

Fig. 2: Circuit layout and die micrograph

At the input of the circuit, a pulsed light source is required with a repetition rate equal to a quarter of the target symbol rate and duty cycle of $25 \%$, i.e. we have pulses with the length of the symbol period of the target signal. The incoming light is then split equally over four parallel branches. In each branch there is an intensity modulator that is driven by electronics operating at a quarter of the aggregate target rate. Next, the branches are recombined after applying respective optical time delays of 0,1,2 and 3 symbol periods to the modulated pulses. As a result, the pulses are interleaved and form a signal at 4 times the rate of the driving electronics. Moreover, the serializer is operating irrespective of the levels in the pulses, so branches can be modulated with both an NRZ and a multilevel format.

An implementation of the proposed structure was fabricated on imec's $200 \mathrm{~mm}$ SiP platform. The layout and a die micrograph are shown in Fig. 2.

A binary tree of multimode interferometers (MMIs) is used to split the light into four branches. Per branch, an $80 \mu \mathrm{m}$ long GeSi EAM modulates the quarter-rate electrical data. The EAM operation is based on the Franz-Keldysh effect, where the bandgap of the device shifts when an electrical field is present. These are the same type of EAMs as used in our previous work $[13,16]$ where we measured an insertion loss (taken as the ratio of the highest signal power level to the average input power) of $\sim 7 \mathrm{~dB}$ and extinction ratio of $\sim 8 \mathrm{~dB}$ for a $2 \mathrm{Vpp}$ swing. As these $\mathrm{GeSi}$ devices are extremely compact, they can be driven as very small capacitors without power hungry resistive terminations. Although EAMs were used in this work, any type of modulator structure can be used as long as it implements an intensity modulation of the signal. This opens up the way to also use other compact and unterminated modulators capable of generating NRZ or PAM4 , such as segmented micro-ring resonators (MRR) [14]. The optical delays on this sample are implemented as waveguide spirals of $\mathrm{N} \times 750 \mu \mathrm{m}$ length $(\mathrm{N}=0,1,2,3)$. Thanks to the high index contrast of the SOI-waveguides, the spirals were realized with a bend radius of $10 \mu \mathrm{m}$, resulting in very compact time delays with very low losses $(<0.2 \mathrm{~dB})$. At 1563 $\mathrm{nm}$, the measured delay difference between each arm is fixed at approximately $9.6 \mathrm{ps}$. This is at the same time the symbol period Ts of the resulting signal (after serializing), therefore making this demonstrator suited for 104 Gbaud operation originating from 26 Gbaud electronics. Finally, the light from each branch is combined with three $2 \times 1$ MMIs and coupled in and out of the photonic IC through fiber-to-chip grating couplers (GC) with an efficiency of approximately -6 $\mathrm{dB} /$ coupler. These could be replaced by more efficient edge couplers $(\sim 1.5 \mathrm{~dB} /$ coupler) to lower the total insertion loss. A drawback of the configuration, however, remains the inherent $6 \mathrm{~dB}$ loss due to combiners after the four branches.

\section{TRANSMISSION EXPERIMENT}

Fig. 3 shows the experimental setup that was used to characterize the fabricated $\mathrm{Si}$ serializer and modulator. To generate a train of optical pulses, CW light at $1563 \mathrm{~nm}$ is launched into a cascaded MZM (biased at minimal transmission and acting as a pulse carver) and a phase modulator (PS) modulated by a $26 \mathrm{GHz}$ sine wave generator. $230 \mathrm{~m}$ of dispersion compensated fiber (DCF) is added to provide additional compression to the pulses, ensuring a FWHM of less than $9.6 \mathrm{ps}$ when measured with a $70 \mathrm{GHz}$ PIN-PD.

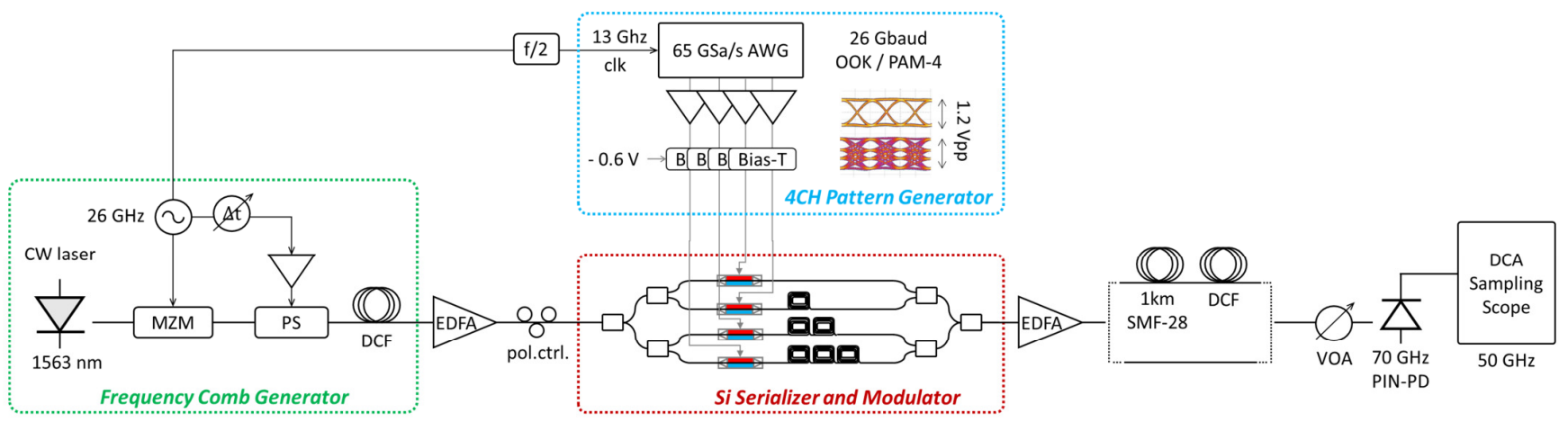

Fig. 3. Experiment setup for $104 \mathrm{~Gb} / \mathrm{s}$ OOK and $208 \mathrm{~Gb} / \mathrm{s}$ PAM-4 transmission with the silicon photonics 4:1 serializer. An MZM pulse carver and a cascaded phase modulator are driven by a $26 \mathrm{GHz}$ sine wave to create a frequency comb from a CW laser. 4 channels from the AWG generate data at 26 GBaud to modulate the pulsed light with NRZ or PAM4 drive signals. The different branches are delayed with spirals waveguides (each $9.6 \mathrm{ps}$ ) to properly interleave the modulated pulses into the final optical waveform with a 104 GBaud symbol rate. 
Inspection of the optical spectrum reveals eleven $26 \mathrm{GHz}$ spaced lines within a $3 \mathrm{~dB}$ flatness (Fig. 4 top). In the time domain signal we can see a pulse periodicity of $38.5 \mathrm{ps}$, corresponding to a repetition rate of $26 \mathrm{GHz}$. Next, an EDFA amplifies the pulses to provide a $13 \mathrm{dBm}$ average input power to the PIC. A four channel $65 \mathrm{GSa} / \mathrm{s}$ arbitrary waveform generator $(A W G)$ creates four uncorrelated data streams to drive the different branches. These are amplified to $1.2 \mathrm{Vpp}$ and applied to the EAMs through two $50 \Omega$ terminated GSGSG RF-probes. All four EAMs were biased at $-0.6 \mathrm{~V}$. After the PIC, an EDFA and variable optical attenuator set the average input power to a $70 \mathrm{GHz}$ PIN-PD $(0.6 \mathrm{~A} / \mathrm{W})$. Finally, the eyes are captured by a $50 \mathrm{GHz}$ sampling oscilloscope to visualize the eye diagrams. For the BER estimations, a 63 $\mathrm{GHz}$ real-time scope is used to capture the waveforms after which the Q-factor is calculated offline. In the experiment, all equipment is synchronized using a $26 \mathrm{GHz}$ clock signal. It is important to have a proper alignment of the pulse carving operation and the AWG data signals to have optimally modulated pulses. When the pulse train generation is replaced by a MLL, active mode-locking would be the natural solution to implement this synchronization. The trigger signal for the MLL and the data signals would then be retimed using the master clock from the CDR typically present in the electronic driver chip.

The average in-fiber power after the PIC is $-14 \mathrm{dBm}$ during operation: $13 \mathrm{dBm}$ (input power) $-6 \mathrm{~dB}$ (grating coupler in) $-7 \mathrm{~dB}$ (IL EAMs) $-2 \mathrm{~dB}(7 \mathrm{~dB}$ dynamic ER at $1.2 \mathrm{Vpp})$ $-6 \mathrm{~dB}$ (4:1 combiner losses) $-6 \mathrm{~dB}$ (grating coupler out). The in-waveguide outer optical modulation amplitude (OMA) after the serializer was $-6.8 \mathrm{dBm}$ for an average in-waveguide power of $-8 \mathrm{dBm}$ (and $+7 \mathrm{dBm}$ in-waveguide power entering the serializer). A considerable part of the loss originates from the $12 \mathrm{~dB}$ insertion loss of the grating couplers. Employing edge couplers $(<2 \mathrm{~dB} /$ coupler) could relieve the total insertion loss and bring the in-fiber power close to $-5 \mathrm{dBm}$. Transmission is tested back-to-back (BtB) and over $1 \mathrm{~km}$ of SMF followed by $150 \mathrm{~m} \mathrm{DCF}(-15.9 \mathrm{ps} / \mathrm{nm})$ to compensate for the accumulated chromatic dispersion at $1563 \mathrm{~nm}$. The data transmission was tested with both NRZ and PAM-4 signals (decorrelated $2^{9}-1$ long pseudo-random bit streams) generated by the AWG at 26 Gbaud.

Fig. 5a shows the captured eye diagrams of the input signals to the modulator as well as the resulting 104 Gbaud OOK and PAM-4 eye diagrams. To capture the individual behavior of each EAM in the eye diagrams, the sampling oscilloscope was triggered with a clock of $1 / 4^{\text {th }}$ of the total baudrate. However, during BER estimations all eyes were superimposed on one period of the total baudrate (104 Gbaud), resembling a conventional full rate optical receiver.. Fig. 5b displays the optical spectrum of the output signal. The lines with a 104 $\mathrm{GHz}$ spacing are emphasized in the frequency comb revealing the $4: 1$ serializing operation. At $104 \mathrm{~Gb} / \mathrm{s}$ OOK, the captured eye diagrams are clearly open, both BtB and after $1 \mathrm{~km}$ of fiber. Based on the Q-factor, we estimate a BER below 7E-7 for BtB and 5E-6 after $1 \mathrm{~km}$ of SMF, without any online or

\section{Frequency domain}

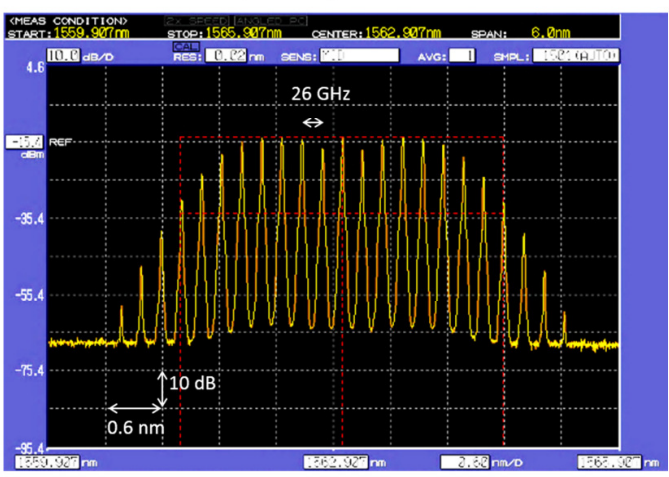

Time domain

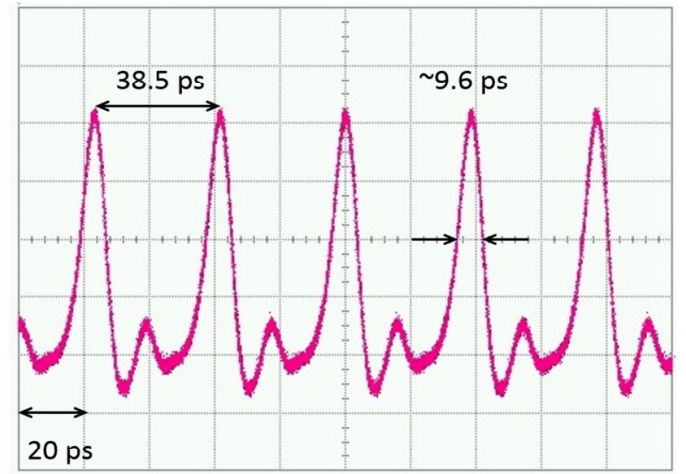

Fig. 4. Frequency domain and time domain plots of the optical input signal to the optical serializer

offline equalization. For PAM-4 transmission, the relative placement of the electrical PAM-4 levels is slightly adjusted in the AWG software to compensate for the compression caused by the non-linear E/O transfer function of the GeSi EAMs. Apart from these adjusted levels, no DSP or equalization is used to generate the $208 \mathrm{~Gb} / \mathrm{s}$ PAM- 4 eyes. Currently, the driving signals for each modulator were not individually optimized. This leads to some unbalancing between the levels of the neighboring tributaries and limits the performance if the signal is received without a demultiplexing operation (as in this experiment). However, a start-up calibration can be implemented to ensure proper alignment, monitoring the optical output generated by a single tributary (placing all other tributaries in a fixed state) and adjusting the driving signal accordingly, and this for all tributaries. Nevertheless, at record speeds of $208 \mathrm{~Gb} / \mathrm{s}$, the different PAM-4 levels are still clearly distinguishable even after $1 \mathrm{~km}$ of SMF. Here, the estimated BERs are $8.9 \mathrm{E}-3$ for a BtB link and 9.9E-3 after $1 \mathrm{~km}$ of SMF, already below the soft decision FEC of $2 \mathrm{E}-2$ (as is commonly used in $200 \mathrm{~Gb} / \mathrm{s}$ coherent transceivers) without using any equalization. Adding pre-emphasis and equalization or the DAC-less parallel EAM topology proposed in [16], could be used to improve these BERs for operation with the current standard intra data center FEC for single-mode transceivers of 2.4E-4 for 200 and $400 \mathrm{GbE}$ transceivers. 

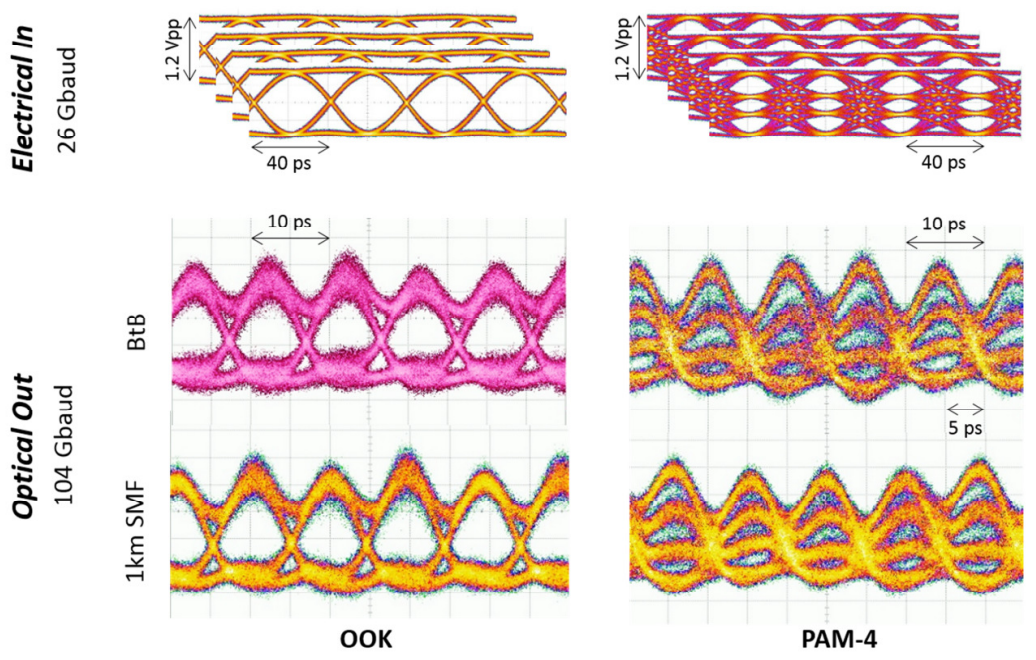

(a)

\section{Spectrum after 4:1 Optical Serializer}

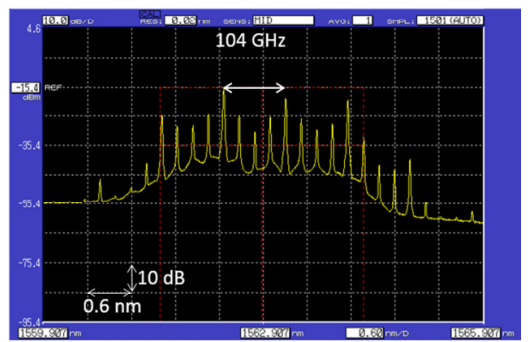

(b)

Fig. 5. (a) Eye diagrams captured by a $50 \mathrm{GHz}$ sampling oscilloscope: 26 Gbaud electrical inputs (OOK/PAM4) and received optical signal in BtB and after $1 \mathrm{~km}$ of SMF at 104 Gbaud OOK and PAM-4 (b) optical spectrum after the optical serializer

\section{DISCUSSION AND PROSPECTS}

In the demonstrated experiment, we have realized the fastest single-wavelength silicon modulator capable of generating PAM-4 and OOK at $104 \mathrm{GBd}$. The EAM modulators in the different branches are very compact and only need to be driven with $1.2 \mathrm{Vpp}$ voltage swing at a quarter of the target symbol rate. As a result, the proposed transmitter topology can be made extremely power efficient. In [15] we have recently shown that for these lumped-load modulators, dedicated drivers can be designed up to $70 \mathrm{~Gb} / \mathrm{s}$ while only consuming $61 \mathrm{~mW}$. When designed for the 26 Gbaud rate in this demonstrator, the drive current could be lowered, hence resulting in even lower power consumption.

However, there is no fundamental hurdle preventing the proposed architecture to operate at higher data rates. The current photonic IC was operated at 26 Gbaud (quarter-rate) since this was the optimal speed for the measured delays of the waveguide spirals. Only a small redesign effort is required to adapt these delays to shorter symbol periods. Alternatively, when not yet limited by the speed of the electronics, one could choose to implement a half-rate structure rather than a quarterrate structures to limit interconnect complexity between electronic and photonic dies and gain $3 \mathrm{~dB}$ in optical power budget.

A next path forward would be to replace the PAM-4 data generation by an alternative topology. Currently, the four-level signal is generated electrically by the AWG and applied to the non-linear electro-optic transfer function of the EAM. Previously we have shown that by moving the DAC functionality to the optical domain drastically improved PAM-4 performance can be obtained with the same EAMs as it bypasses the linearity requirements at the transmitter [16]. In Fig. 6 (a) a waveform is displayed where only one of the
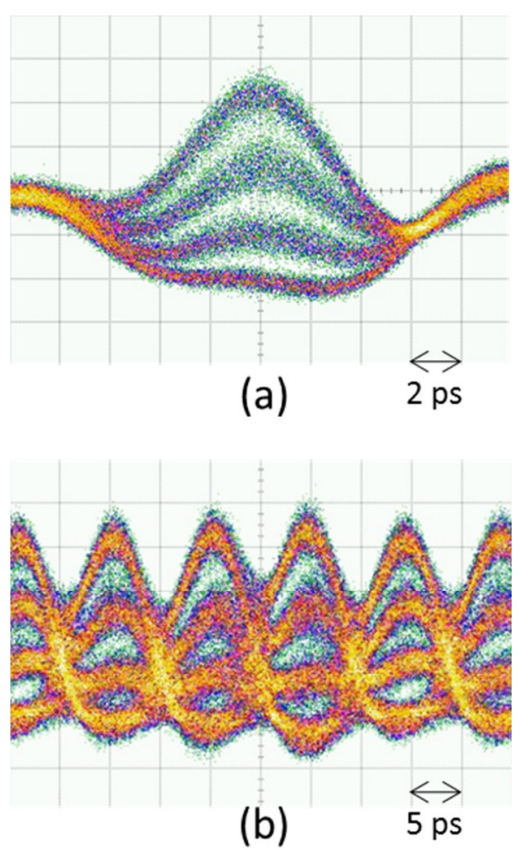

Fig. 6: Waveform where only 1 out of 4 modulators is driven with four-level data (a) and full PAM-4 waveform (b). The waveforms illustrate that the limitation in the PAM4 signal originates from the modulation of the individual pulses rather than from the serializing operation.

branches of the serializer is modulated with multilevel data from the AWG, together with the full PAM-4 waveform. We can see that the limitation for the output signal is already in the modulation of a single pulse and that the serializing adds no visible additional degradation to the levels.

This observation makes it reasonable to assume that employing a better PAM-4 data generation will also result in 
$>10 \mathrm{~nm}$ Mode-Locked Laser
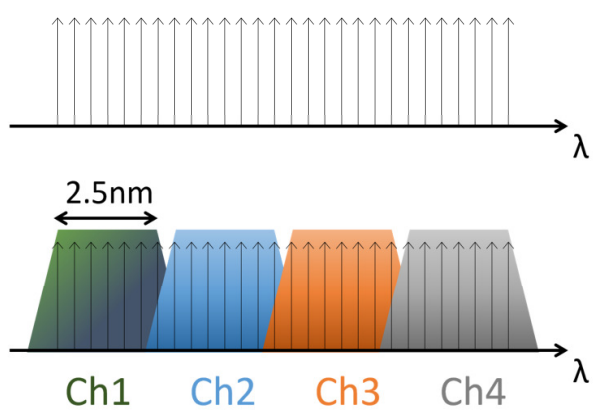

Fig. 7: Illustrating the principle of using a frequency comb as the source of multiple WDM channels. The spectral width could be narrowed down to $2.5 \mathrm{~nm}$ allowing the laser source to be shared over 4 channels.

an improvement when used in the branches of the serializer topology. Ultimately, when combining our 64 Gbaud PAM-4 transmitter demonstrated in [1] with the serializer, it is possible to evolve towards $>0.5 \mathrm{Tbit} / \mathrm{s}$ from a single laser source. Moreover, the alternative PAM-4 transmitter is again suited to be driven with the same electronic IC as in [15]. This would allow us to achieve these record high data rates with an aggregate driver power consumption of only $1 \mathrm{pJ} / \mathrm{bit}$ (excluding the laser).

The need for a pulsed laser source remains a disadvantage compared to a common continuous wave laser source as used in today's optical links. To alleviate this drawback, we investigate the possibility of sharing the laser source over multiple channels. Recent research has illustrated that a frequency comb is a viable alternative as a source for multiple wavelength-division multiplexing (WDM) channels [17]. When expanding our test setup with an optical waveshaper acting as a band pass filter, we could narrow down the spectrum of the frequency comb to less than $2.5 \mathrm{~nm}$ without any observable penalty. As illustrated in Fig. 7, considering a $10 \mathrm{~nm}$ frequency comb, a single laser could be used to feed 4 WDM channels. Exploiting this property of the frequency comb can potentially outweigh the additional hardware complexity of the laser source. Replacing the inefficient grating couplers with edge couplers would already provide sufficient power budget to feed four 200G channels from a single mode locked laser (assuming similar output power of the MLL). Additional margin could be obtained by incorporating heterogeneously integrated SOAs either at the input of the transmitter (providing its spectrum is at least $10 \mathrm{~nm}$ wide) or at the output of each of the four $200 \mathrm{G}$ channels. This way, an $832 \mathrm{~Gb} / \mathrm{s}$ ( 4 × $208 \mathrm{~Gb} / \mathrm{s})$ PAM-4 transmitter based on a single laser could be realized.

\section{CONCLUSION}

We have demonstrated a viable path towards increasing the symbol rate in IMDD links by introducing a 4:1 serializer in the optical domain. Using an implementation based on four GeSi EAMs, we show 104 Gbaud OOK and PAM-4 transmission, realizing the fastest PAM-4 transmitter on a silicon platform. In the future, a single $10 \mathrm{~nm}$ wide MLL could be employed to feed a 4 channel $>800 \mathrm{Gbits} / \mathrm{s}$ system. The data rates can be pushed even further by omitting the electrical multilevel drive signals and incorporating EAM-based PAM4 generation instead.

\section{REFERENCES}

[1] J. Verbist, J. Lambrecht, M. Verplaetse, S. A. Srinivasan, P. De Heyn, T. De Keulenaer, A. Vyncke, M. Vanhoecke, J. Van Campenhout, X. Yin, G. Roelkens, G. Torfs, "First real-time demonstration of $128 \mathrm{~Gb} / \mathrm{s}$ PAM-4 transmission over $1 \mathrm{~km}$ SMF using a Si photonics transmitter", European Conference on Optical Communication, Italy, (2018)

\section{[2] 2018 Ethernet Alliance Roadmap -www.ethernetalliance.com}

[3] B. Moeneclaey, Jochen Verbrugghe, Joris Lambrecht, Elad Mentovich, Paraskevas Bakopoulos, Johan Bauwelinck and Xin Yin, "Design and Experimental Verification of a Transimpedance Amplifier for 64-Gb/s PAM-4 Optical Links," in Journal of Lightwave Technology, vol. 36, no. 2, pp. 195203, Jan 15, 2018.

[4] H. Zhang, B. Zhu, S. Park, C. Doerr, M. Aydinlik, J. Geyer, T. Pfau, G. Pendock, R. Aroca, F. Liu, C. Rasmussen, B. Mikkelsen, P. I. Borel, T. Geisler, R. Jensen, D. W. Peckham, R. Lingle, D. Vaidya, M. F. Yan, P. W. Wisk, and D. J. DiGiovanni, "Real-time transmission of $16 \mathrm{~Tb} / \mathrm{s}$ over $1020 \mathrm{~km}$ using 200Gb/s CFP2-DCO," Opt. Express 26, 6943-6948 (2018)

[5] H. Mardoyan, F. Jorge, O. Ozolins, J. M. Estaran, A. Udalcovs, A. Konczykowska, M. Riet, B. Duval, V. Nodjiadjim, J. Dupuy, X. Pang, U. Westergren, J. Chen, S. Popov, S. Bigo, "204-Gbaud On-Off Keying Transmitter for Inter-Data Center Communications," OFC, San Diego, (2018), PDP-Th4A.4.

[6] J. M. Estarán, H. Mardoyan, F. Jorge, O. Ozolins, A. Udalcovs, A. Konczykowska, M. Riet, B. Duval, V. Nodjiadjim, J. Dupuy, X. Pang, U. Westergren, J. Chen, S. Popov, S. Bigo., "140/180/204-Gbaud OOK Transceiver for Inter- and Intra-Data Center Connectivity," in Journal of Lightwave Technology. Oct 2018 (Early Access)

[7] S. Kanazawa, H. Yamazaki, Y. Nakanishi, Y. Ueda, W. Kobayashi, Y. Muramoto, H. Ishii, H. Sanjoh, "214-Gb/s 4-PAM Operation of Flip-Chip Interconnection EADFB Laser Module," in Journal of Lightwave Technology, vol. 35, no. 3, pp. 418-422, 1 Feb.1, 2017.

[8] E. El-Fiky, M. Chagnon, M. Sowailem, A. Samani, M. Morsy-Osman and D. V. Plant, "168-Gb/s Single Carrier PAM4 Transmission for Intra-Data Center Optical Interconnects," in IEEE Photonics Technology Letters, vol. 29, no. 3, pp. 314-317, 1 Feb.1, 2017

[9] J. Verbist, J. Lambrecht, M. Verplaetse, J. Van Kerrebrouck, A. Srinivasan, P. De Heyn, T. De Keulenaer, X. Yin, J. Van Campenhout, G. Roelkens, J. Bauwelinck, DAC-less and DSP-free PAM 4 Transmitter at 112 $\mathrm{Gb} / \mathrm{s}$ with Two Parallel GeSi Electro-Absorption Modulators, European Conference on Optical Communication (ECOC), Sweden, p.PDP.C.5 (2017)

[10] Thomas Richter, Evarist Palushani, Carsten Schmidt-Langhorst, Reinhold Ludwig, Lutz Molle, Markus Nölle, and Colja Schubert, "Transmission of Single-Channel 16-QAM Data Signals at Terabaud Symbol Rates," J. Lightwave Technol. 30, 504-511 (2012)

[11] Z. Wang et al., "A III-V-on-Si ultra dense comb laser", Light: Science \& Applications, 6, pp. e16260 (2017)

[12] H. Hu, F. Da Ros, M. Pu, F Ye, K. Ingerslev, E. da Silva, Md. Nooruzzaman, Md, Y. Amma, Y. Sasaki, T. Mizuno, Y. Miyamoto, L. Ottaviano, E. Semenova, P. Guan, D. Zibar, M. Galili, K. Yvind, T. Morioka, L. K. Oxenlowe, "Single-source chip-based frequency comb enabling extreme parallel data transmission", Nature Photonics, 12, 2018

[13] J. Verbist, M. Verplaetse, A. Srinivasan, P. De Heyn, T. De Keulenaer, R. Pierco, R. Vaernewyck, A. Vyncke, P. Absil, G. Torfs, X. Yin, G. 
Roelkens, J. Van Campenhout, J. Bauwelinck, First Real-Time 100-Gb/s NRZ-OOK Transmission over $2 \mathrm{~km}$ with a Silicon Photonic ElectroAbsorption Modulator, Optical Fiber Communication Conference (2017), PDP - Th5C.4 (2017)

[14] J. Sun, M. Sakib, J. Driscoll, R. Kumar, H. Jayatilleka, Y. Chetrit, H. Rong, "A 128 Gb/s PAM4 Silicon Microring Modulator," 2018 Optical Fiber Communications Conference and Exposition (OFC), San Diego, CA, 2018, pp. 1-3.

[15] H. Ramon, J. Lambrecht, J. Verbist, M. Vanhoecke, S.A. Srinivasan, P. De Heyn, J. Van Campenhout, P. Ossieur, X. Yin, J. Bauwelinck "70 Gb/s $0.87 \mathrm{pJ} /$ bit GeSi EAM Driver in 55nm SiGe BiCMOS”, European Conference on Optical Communication, Italy, (2018), Tu3E.5

[16] Verbist, Jochem, Joris Lambrecht, Michiel Verplaetse, Joris Van Kerrebrouck, Srinivasan Ashwyn Srinivasan, Peter De Heyn, Timothy De Keulenaer, et al. 2018. "DAC-Less and DSP-Free 112 Gb/s PAM-4 Transmitter Using Two Parallel Electroabsorption Modulators." Journal of Lightwave Technology 36 (5): 1281-1286

[17] Lundberg, L.; Karlsson, M.; Lorences-Riesgo, A.; Mazur, M.; TorresCompany, V.; Schröder, J.; Andrekson, P.A. Frequency Comb-Based WDM Transmission Systems Enabling Joint Signal Processing. Appl. Sci. 2018, 8, 718. 